\title{
Los derechos de la infancia y las políticas públicas. Reflexiones sobre la incidencia del hábitat en los asentamientos de origen informal en Bogotá
}

The Rights of Children and Public Policies. Reflections on the Impact of Habitat on Settlements of Informal Origin in Bogota

Os direitos da infância e as politicas públicas. Reflexões sobre a incidência do habitat nos assentamentos de origem informal em Bogotá

Olga Lucía Ceballos Ramos

Recibido: 11 de julio de 2017

Aprobado: 12 de septiembre de 2017

http://dx.doi.org/10.12804/revistas.urosario.edu.co/territorios/a.5932

Para citar este artículo:

Ceballos, O. L. (2018). Los derechos de la infancia y las políticas públicas. Reflexiones sobre la incidencia del hábitat en los asentamientos de origen informal en Bogotá. Territorios, (38), 167-189. Doi: http://dx.doi. org/10.12804/revistas.urosario.edu.co/territorios/a.5932

* Arquitecta, magíster en Urbanismo de la Universidad Nacional de Colombia, sede Bogotá. Profesora asociada del departamento de Arquitectura de la facultad de Arquitectura y Diseño de la Pontificia Universidad Javeriana. Correo electrónico: olga. ceballos@javeriana.edu. co, ORCID: http://orcid. org/0000-0003-2308-5054 
Palabras clave

Derechos, infancia, politicaspúblicas, asentamientos de origen informal.

Keywords

Rights, childhood, public policies, settlements of informal origin.

Palavras-chave

Direitos, infância, politicas públicas, assentamentos de origem informal.

\section{territarias 38}

\section{RESUMEN}

Las políticas públicas para la infancia en Colombia han tenido un importante avance en este siglo a partir de la promulgación del Código de Infancia y Adolescencia en el año 2006. Con el fin de promover la participación de los niños, niñas y adolescentes (en adelante, NNA) en las decisiones que afectan sus vidas, el Estado ha desarrollado diversas herramientas para la definición de las políticas que deben plantearse en los ámbitos municipal y departamental. No obstante, la participación de los NNA en las decisiones referidas a su hábitat aún no logra transformar algunos factores que limitan el disfrute pleno de sus derechos, especialmente para quienes viven en asentamientos urbanos de origen informal. En este artículo se presentan las reflexiones finales de un estudio exploratorio sobre la incidencia de las condiciones del hábitat en el disfrute pleno de los derechos de los NNA que habitan en asentamientos de origen informal en Bogotá. En este sentido, se hace énfasis en tres condiciones del hábitat que se postulan como factores con un alto impacto negativo: el hacinamiento en la vivienda, la segregación urbana y la estigmatización, todas en relación con las políticas públicas que deberían abordarlas.

\section{AbSTRACT}

Public policies for children in Colombia have made significant progress in this century since the promulgation of the Children and Adolescents Code in 2006. In order to promote the participation of children and teenagers in decisions that affect their lives, the State has developed various tools for the definition of policies at the municipal and departmental levels must be raised. However, the participation of children in decisions regarding their habitat has not yet succeeded in transforming some factors that limit their full enjoyment of their rights, especially for those living in urban settlements of informal origin. This article presents the final reflections of an exploratory study on the incidence of habitat conditions in the full enjoyment of the rights of children living in settlements of informal origin in Bogotá. In this sense, the emphasis is placed on three habitat conditions that are posited as factors with a high negative impact such as overcrowding in housing, urban segregation and stigmatization, in relation to the public policies that should address them.

\section{RESUMO}

As políticas públicas para a infância na Colômbia têm tido um importante avanço neste século a partir da promulgação do Código de Infância e Adolescência no ano 2006. Com o fim de promover a participação das crianças e adolescentes nas decisões que afetam as suas vidas, o Estado tem desenvolvido diversas ferramentas para a definição das políticas que nos âmbitos municipal e departamental devem apresentar-se. No entanto, a participação dos NNA nas decisões referidas ao seu habitat ainda não consegue transformar alguns fatores que limitam o disfrute pleno de seus direitos, especialmente para quem vivem em assentamentos urbanos de origem informal. Neste artigo se apresentam as reflexões finais de um estudo exploratório sobre a incidência das condições do habitat no disfrute pleno dos direitos dos NNA que habitam em assentamentos de origem informal em Bogotá. Neste sentido, se faz ênfase em três condições do habitat que se postulam como fatores com um alto impacto negativo como são a superlotação na vivenda, a segregação urbana e a estigmatização, em relação com as políticas públicas que deveriam abordá-las. 


\section{Introducción}

\section{Derecho a la vida, a la calidad de vida y a un ambiente sano}

Los niños, las niñas y los adolescentes tienen derecho a la vida, a una buena calidad de vida y a un ambiente sano en condiciones de dignidad y goce de todos sus derechos en forma prevalente. La calidad de vida es esencial para su desarrollo integral acorde con la dignidad del ser humano. Este derecho supone la generación de condiciones que les aseguren desde la concepción cuidado, protección, alimentación nutritiva y equilibrada, acceso a los servicios de salud, educación, vestuario adecuado, recreación y vivienda segura dotada de servicios públicos esenciales en un ambiente sano (Colombia, Congreso de la República, 2006, art. 17).

El fenómeno de los asentamientos de origen informal en las ciudades de América Latina se ha convertido en una característica común, derivado de las dificultades de acceso al suelo y, en últimas, del disfrute del derecho a la ciudad que afecta a la población en situación de pobreza. Es así como buena parte de las ciudades ha sido construida sin el cumplimiento de las normas urbanas ni de estándar alguno que defina su calidad.

En Colombia, los asentamientos de origen informal son un fenómeno muy presente en sus principales ciudades, a las que llegan anualmente miles de personas en búsqueda de mejores oportunidades para su vida. Esto en buena medida se ha derivado del conflicto armado interno que durante más de cinco décadas ha generado procesos de desplazamiento forzado, sumado a las dificultades en la economía del país. Para atender esta problemática el Estado ha desarrollado varias políticas, especialmente en materia de la vivienda y del territorio, con el ánimo de lograr una mayor eficiencia en la atención de las demandas habitacionales, pero aún con limitaciones para lograrlo (Ceballos, Caquimbo \& Rincón, 2015).

En Bogotá, buena parte de su territorio ha sido desarrollado por pobladores en situación de pobreza a partir de gestiones a cargo de promotores informales que por iniciativa propia o por encargo promueven la ocupación de suelos no habilitados para el uso urbano. Esto se ha concentrado en sus localidades de periferia, en procesos de integración de suelo rural a urbano u ocupaciones de suelos no aptos, y con el tiempo se han integrado al desarrollo de la ciudad tanto por su expansión como por procesos de regularización (Torres, 2010). Sin embargo, el mejoramiento de las condiciones de habitabilidad para los pobladores que residen en estos asentamientos de origen informal demora muchos años y demanda importantes esfuerzos para alcanzar niveles aceptables de calidad, lo cual no sucede en todos los casos si se consideran los que simplemente no lo logran nunca. En estos contextos de informalidad urbana han nacido y crecido cientos de miles de niños y niñas, quienes en muchos casos han visto limitados sus sueños y posibilidades de un disfrute pleno de sus derechos. territarias 38

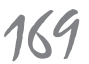


${ }^{1}$ Se realizó un taller con diez madres cuidadoras vinculadas al Programa de Responsabilidad Social Vidas Móviles de la Pontificia Universidad Javeriana; un taller de cuento con niñosy niñas entre los diez y doce años de edad, estudiantes en el Instituto Cerros del Sur, ICES (Barrio Potosí); y entrevistas semiestructuradas con dos lideresas comunitarias y con dos docentes del ICES.

\section{territarias 38} 170
El reconocimiento de esta situación dio origen a un estudio exploratorio de tipo cualitativo sobre la incidencia de las condiciones del hábitat en la infancia, específicamente en cuanto al disfrute de sus derechos en asentamientos urbanos de origen informal. Así se inició, en una primera fase, la revisión del estado del arte sobre los derechos de la infancia y su relación con el hábitat urbano. En la segunda fase se trabajó sobre la percepción de quienes tienen relación con el tema de estudio para ir tejiendo entre lo que dicen los actores involucrados y lo hallado en la revisión del estado del arte. Esta fase se centró en los niños y las niñas hasta la etapa de infancia, en las madres cuidadoras, en las líderes comunitarias y en los docentes del barrio de origen informal Potosí, ubicado en la localidad Ciudad Bolívar de Bogotá. Se propuso como objetivo identificar las condiciones del hábitat que interfieren con el disfrute de sus derechos, para plantear hipótesis de trabajo que serán abordadas en la fase final del estudio que se expone en el presente artículo ${ }^{1}$.

En este sentido, se parte de abordar tres condiciones del hábitat en asentamientos urbanos de origen informal que contribuyen a la vulneración de los derechos de la infancia, frente a las cuales las políticas públicas de infancia y adolescencia, así como de vivienda y territoriales, no han logrado afrontarlas de manera adecuada.

La primera condición es el hacinamiento en la vivienda, muy frecuente en los asentamientos urbanos de origen informal, el cual constituye uno de los factores de la habitabilidad que más vulnera el derecho de la infancia a una vida digna, y es transversal para el cumplimiento de varios derechos. Lo anterior, por cuanto afecta su salud tanto física como mental: en la física, al aumentar la probabilidad de desarrollar problemas respiratorios crónicos, alergias y propagación de infecciones, y en la mental, al elevar el riesgo de depresión y ansiedad, así como de victimización por violencia doméstica y abuso sexual. Por lo tanto, se convierte en un factor que vulnera el derecho a la salud y al cuidado. De igual manera, constituye un factor de desventaja en los procesos de aprendizaje al dificultar la concentración en el momento de estudiar o realizar tareas escolares. Así, vulnera el derecho a la educación (Ceballos, 2016).

La segregación urbana - en el caso específico de los asentamientos urbanos de origen informal - constituye una condición que vulnera el derecho de la infancia al cuidado. Lo anterior porque la localización generalmente alejada de los lugares de empleo para los padres de familia, así como de los servicios urbanos, no les facilita que asuman el cuidado de sus hijos ni el acceso a los servicios de cuidado que brinda el Estado, bien por baja cobertura, por ineficiencia o por baja calidad (Ceballos, 2017).

La violencia simbólica, asociada a la estigmatización de quienes habitan en asentamientos urbanos de origen informal, sumada a la violencia delincuencial, constituye una condición que limita el derecho de la infancia a su autonomía sobre su territorio, al generar temor y desconfianza 
del vecindario. A la vez legitima el uso de la violencia por parte de los mismos habitantes hacia los jóvenes problemáticos, $\mathrm{y}$ perpetúa una situación no solamente violenta hacia los niños sino igualmente desesperanzada hacia el futuro, al ser primero testigos y luego víctimas de la mal llamada “limpieza social” (Ceballos, 2017).

\section{Sobre el hacinamiento de vivienda y el derecho de la infancia a la salud, la educación y el cuidado}

La infancia constituye una etapa fundamental para el desarrollo humano, especialmente la primera infancia. En el caso de América Latina y el Caribe, en donde se tienen amplias desigualdades en las condiciones de vida, se ha identificado que la situación de pobreza afecta con mayor énfasis a los niños y las niñas, respecto a las personas que viven otro ciclo de vida. Asimismo, se tiene que incluso entre la población infantil hay evidentes desigualdades asociadas a factores socioeconómicos, étnicos, de sexo o territoriales, que tienen demostrada incidencia en la mortalidad infantil, especialmente de los niños y niñas en primera infancia (CEPAL, 2016).

El reconocimiento de esta situación ha llevado a que se analicen los diversos aspectos asociados a la pobreza infantil, dentro de los cuales se ha identificado la vivienda como un factor de alto impacto en su desarrollo, en lo cual el hacinamiento se ha reconocido como un factor incidente en la vulneración de los derechos de la infancia. En este apartado se va a abordar esta temática a partir de los estudios sobre pobreza y sobre su impacto en los diferentes derechos de la infancia.

\subsection{Infancia, hacinamiento y pobreza}

En estudios recientes la vivienda ha sido considerada como una de las dimensiones por incluir en metodologías utilizadas para medir la pobreza multidimensional de la infancia. En este sentido, varias de sus condiciones son analizadas en la perspectiva del cumplimiento del derecho a la vivienda digna y adecuada, en donde se tiene en cuenta el hacinamiento como una condición de la habitabilidad importante que se debe analizar.

En este contexto, los estudios relacionados con la pobreza infantil ${ }^{2}$ desde la década de 1990 en Latinoamérica empezaron a reconocer una sobrerrepresentación de la población de NNA en hogares en situación de pobreza por ingresos. Aun cuando hubo una disminución de la cantidad de personas pobres en el año 2000, las magnitudes eran dispares entre los hogares dado que el descenso fue mucho menos significativo en los hogares con NNA, lo que inició el debate sobre la infantilización de la pobreza.

Una fracción muy significativa de este grupo de la población enfrenta adversidades que la perjudican directamente en esta etapa de su ciclo vital, que seguirán teniendo repercusiones negativas a lo largo de su vida y que se transmiten a las generaciones siguientes. La población infantil suele estar atrapada en situaciones de insuficiencia
${ }^{2}$ En el Estado Mundial de la Infancia 2005 se afirma que "Los niños y las niñas que viven en la pobreza (son los que) sufren una privación de los recursos materiales, espirituales $y$ emocionales necesarios para sobrevivir, desarrollarse y prosperar, lo que les impide disfrutar sus derechos, alcanzar su pleno potencialo participar como miembros plenos y en pie de igualdad en la sociedad" (UNICEF, 2005) territarias 38

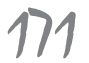


${ }^{3}$ Sorprende, sin embargo, que la de integración geográfica no baya sido considerada una dimensión significativa por el taller de expertos y por ende no se haya incluido, siendo posiblemente la que permitiría aproximarse a establecer la incidencia del territorio en la calidad de vida de los NNA, en tanto que las restantes fueron incluidas en el conjunto de dimensiones por medir, como parte de lo que significa la pobreza infantil multidimensional.

${ }^{4}$ Para la medición de las condiciones de hacinamiento utilizaron como indicadores para niños y niñas de 0-5 años: en privación si habitan en hogares con más de tres personas por cuarto para dormir (excluye baño, cocina, garaje); y para los NNA de 6-17 años: en privación por hacinamiento critico si habitan en hogares con tres o más personas por cuarto para dormir.

${ }^{5}$ En Colombia la Ley 142 define la estratificación en los siguientes términos: "Es la clasificación de los inmuebles residenciales de un municipio, que se hace en atención a los factores $y$ procedimientos que determina la ley" (Departamento Administrativo de Planeación Distrital - DAPD, 2004). Así, en Bogotá, la

\section{territarios 38} 172 de ingreso en los hogares y de privación de los derechos de supervivencia, abrigo, educación, salud y nutrición, entre otros. Es decir que los niños y las niñas se encuentran privados de activos y oportunidades a los que tienen derecho todos los seres humanos (CEPAL, 2013).

Sin embargo, los estudios sobre la pobreza infantil en la región han demostrado que la derivada de privaciones múltiples ha tendido a disminuir en los últimos diez años. Para su análisis, varios países han decidido aumentar el peso de la dimensión de la vivienda en la explicación de la pobreza total en la infancia, encontrando que en general el acceso a viviendas de buena calidad y sin hacinamiento no ha sido suficientemente abordado ni considerado en las políticas de infancia, pese a la importancia que se le reconoce para el adecuado desarrollo de los NNA (CEPAL, 2013).

Ahora, si bien la vivienda es reconocida como el espacio donde los NNA realizan gran parte de las actividades cotidianas fundamentales para su desarrollo, condiciones como el hacinamiento o la precariedad de los materiales de construcción pueden afectarlos. Su autoestima puede verse disminuida, alterando de manera negativa su potencial para contribuir a la sociedad cuando se conviertan en adultos (Tuñón \& González, 2013; Minujin \& Delamonica, 2012). También se reconoce que las condiciones de una vivienda afectan otros aspectos de los NNA como su salud, su privacidad y su habilidad para jugar y hacer sus deberes (Espíndola, Sunkel, Murden \& Milosavljevic, 2017).
En Colombia, un estudio reciente sobre la pobreza infantil demostró que una tercera parte de esta población se encuentra en situación de pobreza múltiple, aun cuando encontró una mejoría entre los años 2008 y 2011 , al pasar del $43 \%$ al $34 \%$. Del estudio se destaca el haber realizado un ejercicio de consulta de la percepción de los NNA para identificar dimensiones constitutivas de la pobreza infantil que no se habían incluido previamente. Como resultado se identificaron las dimensiones de seguridad, integración geográfica, afecto y uso del tiempo, cuidado y recreación ${ }^{3}$. Según los resultados obtenidos, como dimensiones más críticas relacionadas con el hábitat en donde viven se encontraron el hacinamiento ${ }^{4}$, la falta de acceso a zonas verdes y a agua, y el saneamiento (Universidad de los Andes y UNICEF, 2012).

En el caso de Bogotá, en un informe reciente que tomó como base de información la Encuesta Multipropósito 2014, se analizaron las condiciones de la infancia menor de cinco años en situación de pobreza. Los resultados expuestos en el informe no dan cuenta detallada de los diferentes aspectos referidos a las condiciones de habitabilidad, pero señalan que casi la mitad de los niños y niñas en primera infancia habitan en viviendas estrato socioeconómico $2(47,4 \%)$, seguidos por aquellos que habitan en estrato $3(29,9 \%)$. En el estrato 1 habita el $15,1 \%$ y apenas un $5 \%$ habita en el estrato 4 y un $2,7 \%$ en los estratos 5 y 6 (Alcaldía Mayor de Bogotá, 2016) ${ }^{5}$. De conformidad con las condiciones de la habitabilidad previstas en la metodología 
de definición de los estratos socioeconómicos, estos resultados denotan que el $62,5 \%$ de los niños y niñas bogotanos en primera infancia habita en las condiciones más deficitarias de habitabilidad y, en buena medida, en situación de pobreza, aun cuando el informe señala una cifra muy inferior y su disminución entre el 2011 y el 2014: en el 2011 había un 29,9\% de niños y niñas en esta situación, y en el 2014 esa cifra pasó a $26,71 \%$.

Otro informe que hace referencia a la infancia en situación de pobreza en Bogotá, en el cual se incluyen NNA, señala que el $24,46 \%$ de ellos pertenece a hogares pobres por ingresos. Destaca el informe que la pobreza monetaria de la población general de Bogotá fue de 15,76\% en el 2014; la pobreza en hogares con NNA $(24,46 \%)$ fue casi diez puntos porcentuales mayor. La situación es similar en lo referido a la pobreza extrema, pues la general fue de $4,15 \%$ y en hogares con NNA llegó a $4,60 \%{ }^{6}$. En este caso no se hizo el análisis por estratos socioeconómicos, sino que se tomaron las localidades ${ }^{7}$ como unidad geográfica para el análisis, y se encontró que los NNA en situación de pobreza se localizan principalmente en siete de estas: Usme (40,18\%), Ciudad Bolívar (40,11\%), San Cristóbal $(33,46 \%)$, Bosa $(33,03 \%)$, Santa Fe (30,79\%), Rafael Uribe Uribe $(27,02 \%)$ y Kennedy $(25,34 \%)$. Pero sobre el total de Bogotá, la localidad de Ciudad Bolívar es la que concentra el mayor porcentaje: un 17,9\% (Minujin, Bagnoli \& Osorio, 2015).
En ambos informes no se hace referencia a la infancia que habita en condiciones de hacinamiento en la ciudad, pese a contar con datos de la Encuesta Multipropósito 2014 para realizar este análisis, siendo muy probable que las cifras coincidan tanto con los dos estratos socioeconómicos referidos en el primero, como con el hecho de que geográficamente este porcentaje se concentre en su mayoría en asentamientos de origen informal, dado que estos suelen pertenecer a esos dos estratos y predominan en las localidades donde se concentran los hogares pobres con NNA.

\subsection{Hacinamiento, salud, educación y cuidado de la infancia}

En la introducción del presente artículo se ha afirmado que el hacinamiento es una condición de la habitabilidad que vulnera varios derechos de la infancia, $\mathrm{co}^{-}$ mo son el derecho a una vida digna, a la salud, a la educación y al cuidado. Luego, se ha desarrollado una revisión de la incidencia del hacinamiento como factor que permite analizar la pobreza multidimensional con sus diferentes resultados en América Latina, Colombia y Bogotá. En este aparte del documento se argumentará lo concerniente a cada uno de los derechos vulnerados.

En lo referido a la salud, el hacinamiento en la vivienda cuenta con un amplio reconocimiento como factor que la afecta negativamente, tanto en el orden físico como en el mental. En el físico al estar relacionado con problemas respiratorios estratificación es una clasificación de los inmuebles, concretamente los de uso residencial, organizados en seis estratos. Dicha ordenación ascendente implica que las viviendas de peores condiciones de habitabilidad se encuentran en el estrato uno y las de mejor estado, en el estrato seis. La estratificación se compone de los siguientes niveles: estrato 1 : bajo-bajo; estrato 2: bajo; estrato 3: medio-bajo; estrato 4: medio-medio; estrato 5: medio-alto; estrato 6: alto. La estratificación se efectúa teniendo como marco metodológico los lineamientos establecidos para tal fin, mediante la observación de las características físicas exteriormente observables de las viviendas y de su entorno. Para su categorización, en el distrito capital se tienen presentes los siguientes factores, definidos por el Departamento Nacional de Planeación (DNP) a partir de 1997: las características físicas externas de las viviendas, su entorno inmediato y su contexto urbanístico. Teniendo en cuenta estos criterios generales, las variables aplicadas para la conformación de los estratos en el distrito capital son las siguientes: 1. Existencia de entrada principal en las vias del lado de manzana; 2. Vias de acceso; 3. Tamaño
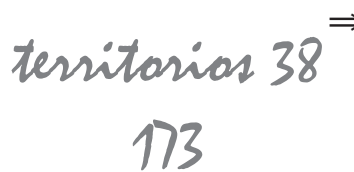
$\Leftarrow$

predominante del frente de las viviendas del lado de manzana; 4. Andén; 5. Antejardin; 6. Garajes; 7. Material de las fachadas; y 8. Material de los techos (Alcaldia Mayor de Bogotá, 2016, p. 12).

${ }^{6}$ Sus datos también se basan en información de la Encuesta Multipropósito 2014 $y$ del Departamento Nacional de Estadistica - DANE.

${ }^{7}$ Las localidades son las unidades político administrativasen las que se divide Bogotá, que en total son veinte, de las cuales una es ruraly las demás son urbanas.

\section{territarias 38} 174 que pueden incluso convertirse en crónicos; y en el mental, en la generación de sentimientos depresivos y de riesgos asociados a la falta de privacidad (Ceballos et al., 2014; Ceballos et al., 2015; Novoa et al., 2014 y Apraez-Villamarín, 2015). En el caso de Bogotá, las estadísticas relacionadas con el abuso sexual de NNA demuestran que sucede con mayor frecuencia en sus viviendas que en los espacios públicos (CODHIA, 2011), lo cual mostraría la importancia de analizar las condiciones de habitabilidad que pueden influir en que esta grave situación de vulneración de derechos suceda en el interior de ellas. Sin embargo, sobre esta problemática se reconocen las limitaciones en los estudios que lo abordan, como lo reconocen Vélez et al. (2015), quienes lo atribuyen al hecho de que suceda principalmente en ambientes privados. Otra problemática que se debe señalar en Bogotá es la del maltrato infantil - el de mayor representatividad en la ciudad-, y el de todos los tipos sucede al interior de los hogares, esto es en sus viviendas (Flórez \& González, 2013). Esto ratifica la importancia de profundizar en el análisis de la incidencia de las condiciones de habitabilidad en el abuso sexual de NNA y en la violencia doméstica, con énfasis en el hacinamiento, pues además de afectar la salud física y mental de los NNA se convierte en una condición inadecuada para su cuidado y salvaguarda de su dignidad humana.

Como se ha señalado, el hacinamiento también afecta negativamente el desarrollo cognitivo de los niños. Se tiene evidencias de asociación entre bajo rendimiento escolar y esta condición, por cuanto no permite la concentración necesaria para el desarrollo adecuado de las tareas escolares (Kaztman, 2011). Asimismo, el no contar con espacios al interior de sus viviendas para el juego ni con parques cercanos limita el desarrollo motriz y de la imaginación de los niños, factores clave para su desarrollo intelectual.

En un estudio desarrollado por Kaztman (2011), en su análisis de la relación entre las privaciones en las condiciones de habitabilidad y el desarrollo del capital humano destaca en sus conclusiones cómo el hacinamiento constituye un factor asociado a la extra edad escolar aun en casos en los que no hay pobreza. Esto demuestra una incidencia directa e independiente de otros factores de una de las condiciones de habitabilidad en la vulneración del derecho de la infancia a la educación. Al igual que lo señalado sobre la importancia de profundizar en el reconocimiento de la incidencia del hacinamiento en la salud y el cuidado de los NNA, los hallazgos de Kaztman enfatizan también la relevancia de considerar esta condición de habitabilidad en el desarrollo del capital humano de la infancia en las políticas públicas pertinentes.

\section{Sobre la segregación urbana, el derecho de la infancia al cuidado y a la educación}

La vida urbana supone una relación del tiempo específicamente ligada a la forma en que está organizado el espacio en 
el territorio. Al respecto, señala Segovia (2016) la importancia de definir una "morfología del tiempo" respecto a horarios y distancias en las diferentes actividades urbanas, para brindar una mejor calidad de vida a las personas y la posibilidad de compatibilizar adecuadamente el trabajo remunerado y el cuidado de los miembros de la familia que lo requieren.

No obstante, la organización del tiempo y del espacio en la ciudad no suele armonizarse en la forma que sugiere Segovia, lo cual afecta negativamente la calidad de vida de las personas que la habitan. Actualmente, en el caso de las ciudades latinoamericanas, el problema no es solamente la ausencia de sincronía entre el tiempo y el espacio urbano, sino que además se presenta cada vez con mayor fuerza la segregación espacial. Según Aymerich, las reformas neoliberales impuestas en los países de la región han aumentado las distancias sociales y han aislado a los pobres urbanos. Señala el zoning como agente que ha propiciado directa o indirectamente segregación, así como la imposición de políticas de planificación urbana para mantener segregaciones socio-espaciales. A lo anterior se suman las políticas de vivienda social que tienden a ubicar los proyectos de vivienda de bajo costo en zonas periféricas de la ciudad para lograr suelo más barato, con lo cual es el mercado el que asume liderazgo en la estructuración del espacio urbano. Aymerich afirma que los pobres urbanos terminan habitando en dos tipos de segregación espacial: unos en la periferia urbana con vivienda y entornos deteriora- dos y otros en áreas centrales también en deterioro (Aymerich, 2004),

Respecto a la situación presente en los asentamientos de origen informal, la reflexión adquiere otro sentido. Si bien desde las políticas públicas este fenómeno urbano ha sido visto como un problema, de otra parte se reconoce como una solución espontánea por parte de la población pobre y excluida frente a la injusticia distributiva (USAID, 1993). Pero al ser parte de un proceso generado de manera diferente a lo que supone el planeamiento urbano y las políticas tanto urbanas como de vivienda, las condiciones espacio-temporales suceden de manera también diferente. El hecho de que estos asentamientos suelan desarrollarse sobre suelos rústicos, y en ocasiones no aptos para la urbanización, ya supone una relación muy diferente con la ciudad construida con base en planes urbanos: están alejados de los centros de servicios urbanos y de empleo, sin infraestructura de servicios públicos, con viviendas construidas de manera precaria. El alcanzar unas condiciones urbanísticamente mejores es parte de un largo proceso de gestión comunitaria. Así, estas condiciones ya definen un entorno que necesariamente va a incidir en la vida de sus habitantes, en cómo se perciben y en cómo se proyectan.

Sobre la incidencia que tienen en la salud y en la educación infantil las condiciones del entorno urbano inmediato en el que habitan los NNA, Kaztman destaca el hecho de que se esté incorporando en modelos explicativos. A su juicio, se trata de una perspectiva que amplía la noción territarias 38

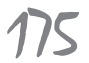


de los ámbitos de socialización primaria al incorporar el vecindario, y demuestra que los roles dominantes en cada una de las generaciones que conviven en un vecindario pueden operar como modelos de comportamiento que afectan las motivaciones y expectativas de los NNA. También, que el nivel de violencia en las calles define la amplitud de las oportunidades con que cuentan los niños para el aprendizaje de la convivencia en la diversidad y para la construcción de capital social (Kaztman, 2011).

Las reflexiones de los autores referidos ponen en evidencia la importancia de las condiciones socio-espaciales en la calidad de vida en general para los pobladores urbanos, pero también llevan a considerar la manera en la cual afectan el disfrute pleno de los derechos de la infancia. La segregación socio-espacial urbana se considera como un agente que aumenta las asimetrías presentes en la calidad del cuidado de la infancia en la ciudad y por ende vulnera este derecho. Ahora, si se tienen en cuenta los asentamientos urbanos de origen informal, en los cuales se concentran en un alto porcentaje hogares en situación de pobreza, generalmente están en desventaja en cuanto al acceso a los lugares de oferta de empleo y a los servicios urbanos, respecto a los hogares que no están en esa situación y habitan en áreas con acceso a ellos. Es por esta razón que las condiciones del vecindario - entre las cuales la localización puede implicar un factor discriminante en la calidad del trabajo y de acceso a la educación - terminan afectando tanto el presente como el futuro de los individuos que lo habitan. Así, la segregación urbana es la expresión espacial de las desigualdades económicas y sociales que genera la concentración de comunidades cada vez más homogéneas, cuyas características acentúan las distancias entre pobres segregados y ricos (Torres, 2016).

Asimismo, la segregación socio espacial afecta el derecho de la infancia al cuidado por dos factores. De una parte, por el tiempo que muchos NNA deben permanecer sin el cuidado de un adulto mientras sus padres trabajan lejos del lugar en donde viven. En la mayoría de los casos en los que el hogar cuenta con un solo jefe o con jefatura compartida, pero que deben trabajar en zonas muy alejadas de sus viviendas, se les dificulta el acceso a los servicios públicos de cuidado de la primera infancia debido a los horarios. En el caso de Bogotá, en los asentamientos de origen informal de la periferia si bien la mayoría de los jardines infantiles públicos prestan sus servicios de 8 am a 5 pm, debido a las largas distancias que deben recorrer los jefes de hogar para llegar a sus lugares de trabajo, se ven obligados a salir desde muy temprano (5 am o $6 \mathrm{am}$ ) y regresar muy tarde ( 7 pm u 8 pm). Esto implica un desfase de tiempo en total de entre cinco y seis horas respecto al horario de los servicios de cuidado (Ceballos, 2017). De otra parte, por problemas de cobertura de los servicios de cuidado de buena calidad, pues no siempre se encuentran cercanos a las viviendas.

Ahora: en relación con el derecho a la educación en Bogotá, el hecho de que 
los asentamientos de origen informal sean segregados a nivel socio-espacial genera dos efectos. En primer lugar, se tienen los problemas de cobertura, pues para muchos pobladores el no tener una escuela pública cercana a sus viviendas se convierte en un obstáculo para el acceso a la educación de los NNA. Esto sucede con mayor frecuencia cuando los asentamientos de origen informal están en etapas iniciales de desarrollo. En segundo lugar, se tiene que el hecho de que las escuelas públicas se localicen en barrios segregados termina también generando segregación en términos de clases sociales e impidiendo un pluriclasismo que permita el encuentro de NNA de diferentes condiciones socioeconómicas que promueva nuevas formas de relación entre ellos y permita superar estigmatizaciones. Esto tiene a su vez dos condiciones, dado que en ello influye tanto la localización en la ciudad como la calidad de la educación, lo que para García y Quiroz (2011) constituyen en Bogotá factores mediante los cuales la educación mantiene la inmovilidad social y la desigualdad.

Según lo expuesto, el hecho de que los asentamientos de origen informal estén segregados social y espacialmente afecta la calidad de vida de sus pobladores al dificultarles su acceso tanto a los servicios de la ciudad como a los lugares de empleo. En el caso específico de los NNA, las condiciones de un entorno urbano segregado les vulneran sus derechos al cuidado y a la educación al dificultar su acceso tanto a los servicios correspondientes como a una buena calidad de los mismos.

\section{Sobre la violencia simbólica y el derecho de la infancia a soñar}

La construcción de estigmas se ha convertido en una expresión de la violencia simbólica, impuesta mediante relaciones de fuerza. No se deriva de un orden natural sino de aspectos culturales, y por tanto es arbitraria y suele aplicarse sobre individuos que ocupan situaciones marginales en la sociedad (Cornejo, 2014). Como elementos que convergen en la generación del estigma para Link y Phelan (2001) se pueden destacar las creencias dominantes sobre lo considerado como indeseable, la discriminación y la pérdida de estatus.

Pero la estigmatización no solamente afecta a determinados individuos o grupos de individuos, sino que igualmente afecta determinados territorios. Como señalan Wacquant, Slater y Pereira (2014), el estigma territorial guarda una estrecha relación con la mancha de pobreza, la subordinación étnica, las viviendas degradadas, las inmoralidades impuestas y la delincuencia callejera. Además, en la actualidad se han nacionalizado y democratizado:

[... en cada país, un pequeño grupo de barrios se ha vuelto universalmente reconocido y atacado a nivel social y espacial por constituirse en refugios donde la indigencia y la decadencia son características que se generan y perpetúan por sí solas. Los nombres de estos barrios sinónimos de infiernos sociales circulan tanto en discursos periodísticos, políticos y académicos como en conversaciones cotidianas. [...] los territarias 38 177 
barrios estigmatizados y desfavorecidos de la ciudad postindustrial suscitan una cantidad abrumadora de emociones negativas y reacciones severas y correctivas impulsadas por miedo, repulsión y rechazo, lo que a su vez fomenta el crecimiento y glorificación de la faceta penal del Estado, de modo de castigar este tipo de marginalidad urbana (Wacquant, Slater \& Pereira, 2014).

Para Molina (2015), en cuanto al tipo de población que más afecta la estigmatización es la de los jóvenes, y en lo territorial a las zonas urbanas en donde predominan personas en situación de pobreza, entre ellos los asentamientos de origen informal. En cuanto a la estigmatización de los jóvenes, se tienen las crónicas policiales con la mención de NNA como actores en hechos violentos, caracterizando a la delincuencia como profesión. Así se les descontextualiza como seres humanos y como NNA sujetos de derechos. Pero también señala Molina que en general a los pobres se les asocia con valores negativos como la vagancia, los vicios, las enfermedades mentales, la violencia y la delincuencia. Respecto a la estigmatización territorial se equiparan los barrios pobres, en su mayoría de origen informal, como los lugares de la delincuencia. Así, tanto con la criminalización de la pobreza como con la del espacio urbano en donde habitan los pobres, se les vulneran sus derechos ciudadanos al trabajo, a la salud y a la educación. Las representaciones mediáticas que se hacen de

\section{territarias 38} los asentamientos de origen informal van generando una imagen negativa que puede legitimar el perpetuar su marginalidad.

En el caso de Bogotá, la estigmatización de personas y de territorios se ha concentrado principalmente en su área periférica, en donde también se encuentra un alto número de asentamientos urbanos de origen informal. Allí convergen tanto la estigmatización externa como la interna:

[...] la paulatina militarización y paramilitarización de espacios públicos en las localidades de Bosa, Ciudad Bolívar, Usme, San Cristóbal, Suba, Kennedy y Usaquén, entre otras, agudiza sus tenazas. Con los 'toques de queda', de los paramilitares dicen que responden al llamado de las comunidades ante los altos índices de inseguridad, vicio y pandillismo, por lo cual ellos llegan a imponer el orden. En verdad, imposición de miedo y terror, y, por su conducto, extensión del control político (Carreño, 2009, s. p.).

Carreño hace referencia a la estigmatización interna, por parte de otros habitantes de estos territorios hacia los jóvenes que conduce a legitimar que determinados grupos armados reconocidos como de derecha realicen acciones violentas en su contra, pero en las que subyacen intereses políticos de dominio territorial (Gómez, 2013). La situación fue especialmente intensa en la década de 1990 en la localidad de Ciudad Bolívar, al punto de contabilizar más de 300 jóvenes asesinados en el lapso de un año (Forero \& Molano 2015).

Estas circunstancias de violencia delincuencial y simbólica suman a la estigmati- 
zación doble que se ha señalado, dado que no solamente los pobladores de la "ciudad formal" estigmatizan a los pobres que habitan en zonas urbanas degradadas, sino que de igual manera los mismos habitantes de estos barrios estigmatizan a los jóvenes y se legitiman acciones violentas en su contra.

Retomando lo señalado líneas atrás en cuanto a la incidencia de las condiciones del entorno físico espacial en el desarrollo de la vida de sus pobladores y en las circunstancias en las cuales los NNA aprenden a socializar y a construir comunidad, la estigmatización interna y externa se convierte en un agente que incrementa el miedo y limita sus sueños (Ceballos, 2017). Su mirada hacia el futuro es de incertidumbre y desconfianza, lo cual vulnera un derecho fundamental de todo ser humano: el de soñar y proyectarse hacia un futuro feliz.

\section{Políticas públicas de vivienda y territorio, y los derechos de la infancia: ¿̨historia de un desencuentro?}

En América Latina y el Caribe se reconoce el avance reciente en el mejoramiento de las condiciones de vida para la infancia. Las cifras indican la disminución de la probabilidad de que niños y niñas mueran en el parto o en sus primeros años, y en general sus condiciones nutricionales han mejorado. Sin embargo, aún se registran retrasos en áreas críticas como el lenguaje y las capacidades cognitivas. Según los cálculos realizados por el Banco Interamericano de Desarrollo - BID, los gobiernos de la región invierten más recursos en la infancia que en la primera infancia, aun cuando es más efectivo para un mejor desarrollo invertir en la primera infancia:

[...] el gasto per cápita de los gobiernos es tres veces superior en niños de entre 6 y 11 años que entre los que tienen entre 0 y 5 años. Además, las inversiones en la primera infancia tienden a estar concentradas de manera desproporcionada en infraestructura física, por ejemplo: en las instalaciones de los jardines de cuidado infantil, y se invierte menos en la capacitación y en el capital humano de los que las atienden. Las más recientes investigaciones demuestran que los retornos más altos de las inversiones pueden provenir de programas modestos que se concentren en mejorar las primeras interacciones cruciales entre los niños y los adultos, ya sean padres, maestros o cuidadores (Moreno, 2015, p. 12).

Sin embargo, al ser evidente que la problemática de la pobreza infantil en la región no solamente afecta a los NNA sino que de igual manera impacta negativamente en la sociedad en su conjunto, se aboga por políticas mediante las cuales se protejan sus derechos. En este sentido se reitera la importancia de priorizar acciones articuladas desde los diferentes sectores, a lo que la CEPAL plantea la necesidad de impulsar los derechos de los NNA no solamente desde la esfera privada sino fundamentalmente desde el Estado, mediante las políticas públicas pertinentes (CEPAL, 2013) territarias 38 179 
En el marco del estudio desarrollado, de cuyos resultados da cuenta el presente artículo, es necesario revisar hasta qué punto las políticas públicas en Colombia se articulan y tienen presentes las condiciones del hábitat en donde viven los NNA en la protección de sus derechos. Para el efecto se expone a continuación un análisis de las políticas territoriales y de vivienda con el objetivo de identificar sus alcances y perspectivas en la defensa de los derechos de los NNA, haciendo énfasis en Bogotá y en la problemática de los asentamientos de origen informal.

\section{Vivienda y territorio. Una mirada desde los derechos de la infancia}

La ciudad colombiana se ha caracterizado por el rápido crecimiento experimentado desde el siglo anterior, explicado en buena medida por las políticas orientadas más al desarrollo urbano que al rural. Lo anterior ha pretendido el logro de un mayor crecimiento económico, pero de otra parte el conflicto armado interno ha sido un agente importante en este fenómeno dado que ha desplazado a millones de personas durante más de medio siglo. Esto explica en buena medida la concentración del $75 \%$ de la población total en las ciudades, en lo cual no ha habido una adecuada respuesta de las administraciones locales desbordadas en su capacidad por el acelerado proceso. Por esta razón, un importante porcentaje de la población de migrantes termina en situación de pobreza, así como también se ven afectados por problemas relacionados con la calidad del ambiente construido y por las dificultades para acceder a una vivienda adecuada (Ceballos, Caquimbo \& Rincón, 2015).

En este contexto, en la definición de las políticas de vivienda y desarrollo urbano en el país desde su inicio a comienzos del siglo anterior, el Estado ha desempeñado un papel en el que ha priorizado lógicas económicas más que socio-ambientales, aun cuando al menos durante buena parte del siglo XX su responsabilidad sobre lo público estuvo centrada en invertir con un enfoque de bienestar. Este enfoque cambió sustancialmente con la adopción del modelo económico neoliberal, que alteró significativamente la noción de Estado y el rol de lo público en la estructura social en Colombia. Pero una lectura general de sus políticas permite poner en evidencia que sus propuestas se han centrado más en el logro del crecimiento económico en beneficio de un sector particular de la población que en alcanzar una distribución equitativa de la riqueza (Ceballos, Caquimbo \& Rincón, 2015).

El problema de la vivienda, si bien ha constituido una preocupación permanente en la política pública colombiana desde su inicio, no ha sido el tema prioritario. Por esto, no ha sido posible el diseño y la puesta en marcha de una política nacional, permanente e integral de Estado, que logre atender efectivamente las demandas acumuladas existentes en Colombia. Lo anterior deja en evidencia la reducción del tema de la vivienda a los problemas 
económicos relacionados con los sistemas de financiamiento y las políticas de asignación de subsidios. Asimismo, es evidente en la ausencia de la regulación de la calidad de su producción, tanto en la escala arquitectónica como en la territorial (Torres, 2010). Esto se debe en buena medida al cambio en la política de vivienda desde el año 1991, con la promulgación de la Ley $3 .^{a}$ (Congreso de la República, 1991), mediante la cual se adoptó el sistema del subsidio a la demanda como principal instrumento de política ${ }^{8}$. Aun cuando el artículo 51 de la Constitución Política promulga el derecho de todos los colombianos a una vivienda digna (República de Colombia, 1991), son limitadas las normativas que definen su calidad, por lo cual es el mercado el que termina definiendo las condiciones de habitabilidad (Tarchópulos \& Ceballos, 2003).

Así, se continúa privilegiando la atención del déficit cuantitativo en un esquema donde lo prioritario es sobrellevar los ciclos negativos del sector de la construcción para minimizar su impacto en la economía nacional. En el caso de Bogotá, esto ha dado como resultado proyectos de vivienda inadecuados a las necesidades de los hogares, en donde se continúan presentando problemas de hacinamiento y falta de privacidad, entre otras deficiencias (Tarchópulos \& Ceballos, 2003).

Por lo expuesto, la vivienda dirigida a los sectores de menores ingresos presenta deficiencias: su espacialidad no es insuficiente solamente en su interior, sino que de igual manera en su entorno se presentan condiciones deficitarias en cuanto al espacio público y los equipamientos. Como se indicó previamente, la ausencia de normas que demanden unas condiciones mínimas de calidad para la vivienda deja en manos del mercado definir las características de la oferta. En este sentido, los promotores de vivienda defienden la rentabilidad de sus proyectos $y$, considerando que por ley se debe cumplir con un precio tope para la vivienda social, lo logran desmejorando el producto en cuanto a disminución de áreas y acabados de construcción (Tarchópulos \& Ceballos, 2003). Consideraciones como adecuación a las necesidades de los hogares y a aspectos culturales particulares son ignoradas por los promotores formales de vivienda.

Entonces no sorprende que se cuente con una guía de "calidad de la vivienda de interés social" —que obviamente no es de obligatorio cumplimiento- en la que se definen condiciones de calidad que no son exigibles y además se plantean estándares revaluados. Ejemplo de esto es reconocer los efectos nocivos para la salud humana que significa el hacinamiento y aun así proponer estándares inadecuados. Estas contradicciones se ven en los siguientes apartados:

2.1.1. La vida marital de la pareja. La vida marital exige a que la vivienda contar con un dormitorio independiente para la pareja, el cual pueden compartir solo con los hijos menores de 3 años (cuando los haya).
${ }^{8}$ La vivienda social se define a partir de su valor comercial, con un máximo de 135 salarios minimos legales vigentes. Para acceder alsubsidio los hogares deben contar con capacidad de aborro $y$ de endeudamiento en el sistema financiero para tener una solución a su problema habitacional, lo cual es inviable para los más pobres. territarias 38

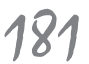


${ }^{9}$ En el caso de la oferta formal mediante el aumento de la densidad de viviendas por hectárea, facilitado por la normativa urbana y la reducción de áreas y acabados. En el caso de la oferta informal, con la entrega de lotes sin obras de urbanismo ni servicios públicos, mediante una acción en la cual los promotores se quedan con toda la plusvalia generada por su intervención (Tarchópulos \& Ceballos, 2005).

\section{territarias 38} 182
2.1.2. El género de los miembros del hogar. Teniendo en cuenta que, independientemente del grupo etario al cual pertenezca la persona: adulto, joven o niño, la vivienda debe disponer de un dormitorio separado para cada género con capacidad máxima de 3 personas por habitación (énfasis añadido).

[... El hacinamiento y el aire contaminado en el interior de la vivienda propician, entre otras, las siguientes enfermedades: tuberculosis, infecciones respiratorias agudas (influenza, neumonía, rinitis, laringitis), asma y lepra.

[... Los padres deben dormir en un espacio diferente de las habitaciones de los hijos. Los hijos pueden dormir juntos si pertenecen al mismo género, niños con niños y niñas con niñas. Algunos problemas como la violencia y el abuso sexual infantil están asociados al hacinamiento (Ministerio de Ambiente, Vivienda y Desarrollo Territorial, 2011 , s. p.).

Se llama la atención en el hecho de admitir tres personas como cantidad máxima por habitación, considerando el estudio sobre pobreza infantil realizado en el país, y referido líneas atrás, en el cual se tomaron los siguientes indicadores para la medición de las condiciones de hacinamiento: para los NNA de 6-17 años, en privación por hacinamiento crítico si habitan en hogares con tres o más personas por cuarto para dormir (Universidad de los Andes y UNICEF, 2012).

Ahora bien, si esto sucede en la oferta formal de vivienda, en el caso de los pro- motores informales, quienes finalmente atienden a la demanda de vivienda de los más pobres, en donde por razones obvias no se cumplen parámetros de calidad, la situación es aun más dramática. Lo que resulta paradójico es que dos tipos de oferta antagónicos como la formal y la informal registren cierta similitud en cuanto a deficiencias de calidad, como se ha señalado en el caso de Bogotá (Tarchópulos \& Ceballos, 2003). La explicación de este fenómeno se encuentra en el hecho de que tanto la oferta formal como la informal persiguen el logro de la mayor rentabilidad posible, $\mathrm{y}$ ante la ausencia de mayores regulaciones de la calidad es posible entender por qué sus resultados en este aspecto los acercan'.

Esto explica en buena medida los resultados hallados tanto en la literatura como en campo sobre la incidencia de las condiciones del hábitat en los asentamientos de origen informal en la vulneración los derechos de la infancia. No es suficiente definir equipamientos para el cuidado, la salud y la educación, sino que es igualmente importante revisar desde el interior de la vivienda hasta su entorno las condiciones por mejorar para salvaguardar sus derechos.

\section{Ciudades prósperas: ¿una alternativa para el encuentro entre políticas territoriales y de infancia?}

Una propuesta interesante en procura del encuentro entre las políticas territoriales y las de infancia ha sido la liderada recientemente por el Instituto Colombiano de 
Bienestar Familiar (ICBF) mediante el proyecto denominado "Ciudades prósperas de los niños, niñas y adolescentes". De manera conjunta con 24 administraciones municipales de la misma cantidad de ciudades del país, en el año 2012 promovieron esta iniciativa con el objetivo de lograr acciones y proyectos interagenciales que permitieran un impacto positivo en la calidad de vida de los NNA en sus territorios. Como uno de sus siete ejes temáticos se incluyó el denominado Desarrollo Urbano Incluyente, dirigido a promover la participación de los NNA en el diseño urbano, con especial atención en el espacio público. Resulta interesante que en esta propuesta se reconoce la baja participación de la ciudadanía sobre las decisiones referidas al desarrollo de la ciudad y al espacio público, siendo casi nula en el caso de los NNA. Por esto incluyeron en la metodología las fases de diagnóstico, anteproyecto, proyecto, construcción o adecuación.

Esta iniciativa se acoge en buena medida a lo pretendido por el programa de UNICEF desarrollado en varios países, conocido como Ciudades Amigas de la Infancia. Esta iniciativa internacional desde 1996 ha venido trabajando para velar por el cumplimiento de la resolución aprobada durante la Segunda Conferencia de las Naciones Unidas sobre Asentamientos Humanos (Hábitat II), cuyo objetivo era lograr ciudades donde todos pudieran vivir. En esta conferencia se postuló el bienestar de los niños como indicador definitivo de un hábitat saludable, de una sociedad democrática y de una buena gobernanza (UNICEF, 2009).

Pero el avance de este tipo de iniciativas entra en conflicto con los intereses de los adultos, los cuales terminan por imponerse: es más importante un buen funcionamiento del tráfico automotor que la posibilidad de que los niños disfruten la calle (Sanz, 2014).

En este sentido una iniciativa que ha tenido el propósito de generar espacios de participación democrática de la infancia sobre las decisiones que afectan su hábitat, según los planteamientos de Tonucci (2005) y Hart (1993), termina limitado a la definición de programas dirigidos a ella, pero sin consultarla. Esto es lo que ha sucedido en Bogotá, pues al consultar sobre los avances de este programa en la ciudad se ha obtenido información detallada de los programas propuestos para la infancia en la ciudad, pero queda claro que no ha habido participación de los NNA, O al menos esta ha sido muy limitada ${ }^{10}$. Se continúa planteando programas basados en diagnósticos sectoriales, sin duda relevantes, pero sin escuchar la voz de los NNA, lo cual sería especialmente importante en los asentamientos de origen informal, dado que es allí en donde se concentran las mayores problemáticas de pobreza.

No obstante, un programa como el de Ciudades Prósperas puede ser la vía para el inicio de una nueva manera de pensar la ciudad desde los territorios y desde las personas, siendo aun más importante la participación de los NNA para una defensa
${ }^{10}$ El 8 de junio del 2017 se recibió respuesta a la consulta sobre los avances del programa por parte de Daniel Monsalve Ortiz, director de Planes de Desarrollo y Fortalecimiento Local de la Secretaría Distrital de Planeación de Bogotá. En su extensa y detallada información (donde explica datos de diagnóstico, responsabilidades según sector y metas), no bay alusión a procesos participativos con los NNA. Tampoco se aluden acciones sobre el espacio público, lo cual es de vital importancia en los barrios de origen informal. territarios 38

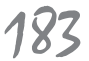


integral de sus derechos. El problema no es solamente revisar las condiciones del espacio público circunscrito a una escala barrial, sino que la reflexión debe ampliarse a cómo se piensa y construye la ciudad en su totalidad y cómo se integran acciones para superar factores como el hacinamiento en la vivienda, la segregación urbana y la estigmatización territorial y social.

\section{Conclusiones}

En este artículo se han expuesto los efectos nocivos de tres condiciones del hábitat que inciden en la vulneración de los derechos de los NNA, y se han revisado respecto a los enfoques de política pública sobre la vivienda y el territorio.

La primera condición del hábitat asociada a la vulneración de varios derechos de la infancia es el hacinamiento en la vivienda, la cual tiene una alta presencia en los asentamientos de origen informal. Como ha sido demostrado en una importante cantidad de estudios, el hacinamiento es un factor negativo en la calidad de vida de las personas que deben habitar en viviendas con esta condición, dado que les afecta su salud física y mental. En el caso específico de los NNA, las evidencias analizadas demuestran que es una condición que vulnera sus derechos al cuidado, a la salud y a la educación, dada su asociación con problemas de salud respiratoria, depresión, violencia intrafamiliar, abuso sexual, así como con algunas dificultades en los procesos de aprendizaje. Por lo tanto, se resalta como una condición del hábitat que debería ser intervenida desde las políticas públicas de vivienda para garantizar la salvaguarda de los derechos de los NNA que vulnera.

La segunda condición del hábitat que vulnera los derechos de los NNA que habitan en asentamientos de origen informal es la segregación urbana. Como se ha establecido en el estudio desarrollado, el hecho de habitar en barrios de origen informal localizados en la periferia urbana, en condiciones de servicios urbanos, de conectividad y de oferta de empleo deficitarios, termina afectando negativamente las posibilidades de cuidado por parte de los padres o tutores de los NNA a su cargo. Lo anterior, por cuanto en numerosos casos deben desplazarse grandes distancias que ocupan una cantidad de tiempo importante, sumado al tiempo que laboran. Esta situación, también en muchos casos, no es posible resolverla mediante el uso de los servicios públicos de cuidado para la primera infancia, considerando los horarios de estos que no cubren el tiempo que se necesitaría. Igual sucede con los demás rangos de edad, pues los colegios generalmente ofrecen media jornada, lo cual deja una buena parte del día a los NNA sin cuidado por parte de un adulto, cuando los padres o tutores no cuentan con una red de apoyo en su cuidado durante su ausencia en el hogar. En consecuencia se afirma que esta condición vulnera el derecho de los NNA al cuidado. De igual manera, la segregación conduce a una cobertura ineficiente del servicio de educación, lo cual afecta negativamente a muchos NNA y dificulta 
su acceso a este. Se resalta también lo que implica la segregación socio espacial, al evitar el encuentro entre diversos grupos sociales en los colegios públicos y fomentar de alguna manera la estigmatización social y territorial. Así, se señala como una condición que debería ser abordada desde las políticas de planeamiento territorial para garantizar los derechos de los NNA al cuidado y a la educación, en iguales condiciones de calidad a quienes habitan en otras áreas de la ciudad.

La última condición del hábitat señalada como vulneradora de los derechos de los NNA que habitan en asentamientos de origen informal es la estigmatización social y territorial. La social, por cuanto los grupos de poder señalan a los pobres y en general a los que habitan en zonas urbanas caracterizadas por la pobreza, como delincuentes, enfermos mentales, consumidores de drogas, entre otros. La territorial, en cuanto los grupos de poder señalan las partes degradadas de la ciudad, bien sea por su origen o por abandono, como lugares peligrosos e indeseables. Esto afecta especialmente a los jóvenes, quienes en el caso de Bogotá, y especialmente en la localidad de Ciudad Bolívar, han sido víctimas de asesinatos justificados por cierto sentido de orden social. Lo preocupante es que esto se ha convertido también en una forma de estigmatización al interior de barrios desacreditados, donde algunos de sus habitantes se sienten con el derecho de decidir sobre la vida de quienes consideran indeseables para la sociedad. Esto afecta muy gravemente el derecho de los NNA a una vida digna, pues en muchos casos limita sus sueños de un futuro diferente al mundo de la pobreza, cuando son no solo víctimas sino, igualmente, testigos de estos hechos violentos. Les limita también en su autonomía para disfrutar libremente su territorio y les lleva a una mirada pesimista y desconfiada de la vida misma. Por lo tanto, esta es otra condición que debería ser trabajada en las políticas territoriales y sociales para garantizar los derechos de los NNA a una vida digna.

De conformidad con lo expuesto, al considerar los derechos de los NNA, y específicamente lo estipulado en el artículo 17 del Código de Infancia y Adolescencia (Congreso de la República, 2006) respecto a "derecho a la vida, a una buena calidad de vida y a un ambiente sano en condiciones de dignidad y goce de todos sus derechos en forma prevalente", la persistencia de las condiciones del hábitat señaladas en el presente artículo debería constituirse en prioridad para las políticas de infancia, vivienda y territorio. No obstante, se ha encontrado que no ha existido un interés en afrontarlas de manera decidida. Es evidente la ausencia de políticas territoriales y de vivienda que incluyan estándares y acciones para corregirlo. Una acción pertinente sería considerar varios aspectos como los efectos del vecindario en la vida de sus pobladores, en la procura de promover desarrollos más equilibrados para la reducción de la desigualdad, con beneficio especialmente para los NNA. Es lamentable que aún la lógica de la integración social no resulte importante para la estructuración y territarias 38

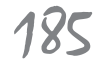


ejecución de los principales programas de las políticas territoriales y de vivienda. Otra acción importante sería la de definir un estándar específico en cuanto a la cantidad de personas por cuarto para dormir, limitándolo a máximo dos del mismo género y rango de edad, salvo el caso de parejas conyugales. El que se mantenga en la guía para la calidad de la Vivienda de Interés Social (VIS) un estándar de hasta tres personas por cuarto para dormir (Ministerio de Ambiente, Vivienda y Desarrollo Territorial, 2011) va en contravía de los estándares internacionales y sostiene la vulneración de derechos de los NNA, denunciada en el presente artículo.

Aunque en Colombia, de manera similar a lo que se registra en América Latina y el Caribe, se reconoce el esfuerzo al igual que los logros por superar varios de los factores que vulneran los derechos de los NNA, debe señalarse que ha faltado definirlo con un enfoque real de derechos en la procura de alcanzar acciones integrales y no sectoriales. Esto significaría recuperar las políticas universales que defiendan el ejercicio integral de los derechos ciudadanos (Pautassi \& Royo, 2012). Además porque la disociación entre las políticas públicas conduce a una seria dificultad para la superación de las problemáticas que más afectan a los NNA. En este contexto es posible afirmar que el mejoramiento de las condiciones de la infancia en general, y en particular de la defensa del disfrute pleno de sus derechos, será viable cuando las políticas territoriales, de vivienda y de infancia asuman un enfoque integral de derechos, en el cual se prevean las circunstancias específicas de las diferentes formas de habitar el territorio y de percibirlo, en procura de la superación de las asimetrías que persisten en la ciudad.

\section{Referencias}

Alcaldía Mayor de Bogotá (2016). Condiciones de la primera infancia en Bogotá, 2011-2014.

Apraez-Villamarín, G. (2015). Factores de riesgo de abuso sexual infantil. Colombia Forense, 2(1), 87-94. doi: http:// dx.doi.org/10.16925/cf.v3il.1179

Aymerich, J. (2004). Segregación urbana y políticas públicas con especial referencia a América Latina. Revista de sociología, 18, 117-130.

Carreño, J. (2009). Estigmatización, persecución y muerte juvenil: Bogotá, jóvenes. Recuperado de https://www. desdeabajo.info/ediciones/3911e s t i g m a ti z a c i \% C $3 \%$ B 3 n persecuci\%C3\%B3n-y-muerte-juvenilbogot\%C3\%Al-j\%C3\%B3venes.html

Ceballos, O. (2016). Infancias, vivienda $y$ ciudad. Reflexiones en el contexto de Bogotá, Colombia [inédito].

Ceballos, O. (2017). Derechos de la infancia $y$ hábitat urbano informal en Bogotá. Una lectura desde el territorio [inédito].

Ceballos, O., Caquimbo, S. \& Rincón, M. (2015). La ciudad informal y la planeación del desarrollo en Colombia. En Desarrollo, prácticas y discursos emergentes en América Latina. ClACSO.

Olga lucía Ceballos Ramos 
Ceballos, O., Caicedo, J., Fernández, A. \& Rincón, M. (2014). Salubridad de la vivienda informal. En El traspatio de la ciudad. Desafíos, prácticas y recomendaciones de politica pública y de capacitación frente a la vivienda informal. Bogotá: Swisscontact, Fundación Suiza de Cooperación para el Desarrollo Técnico.

Ceballos, O., Fernández, A., Giraldo, C., Rincón, M., Londoño, O., Chaparro, P., Macías, F., Martínez, J., Caicedo, J., Montenegro, G., Vega, R. \& Herrera, R. (2015). La habitabilidad de la vivienda y la salud en Colombia. Una propuesta metodológica comprensiva para su análisis. Revista bitácora urbano territorial, $1(25)$.

Cepal (2013). Pobreza infantil en América Latina y el Caribe. En Panorama social de América Latina 2013. Santiago de Chile: CEPAL.

CEPAl (2016). Panorama social de América Latina 2016 [documento informativo]. Santiago de Chile: CEPAL.

CODIA - Comité Operativo Distrital de Infancia y Adolescencia (2011). La politica de infancia y adolescencia en Bogotá D.C. 2011-2021. Bogotá: CODIA.

Colombia, Congreso de la República. Ley 1098 de 2006. Código de la infancia y la adolescencia.

Cornejo, C. (2014). Estigma territorial como forma de violencia barrial. El caso del sector 'El Castillo'. Revista INVI, 27(76), 177-200.

Espíndola, E., Sunkel, G., Murden, A. \& Milosavljevic, V. (2017). Medición mul- tidimensional de la pobreza infantil. CEPAL UNICEF.

Forero Hidalgo, J., \& Molano Camargo, F. (2015). El paro cívico de octubre de 1993 en Ciudad Bolívar (Bogotá): la formación de un campo de protesta urbana. Anuario Colombiano de Historia Socialy de la Cultura, 42(1), 115-143. doi:https://doi.org/10.15446/achsc. $\mathrm{v} 42 \mathrm{nl} .51347$

Flórez, M. \& González, A. (enero-junio, 2013). Caracterización de la violencia intrafamiliar, maltrato infantil y abuso sexual en Bogotá D. C. durante el año 2011. Teoria y praxis investigativa, $8(1), 74-91$.

Gómez, N. (2013). Partir de lo que somos. Alcaldía Mayor de Bogotá, Secretaría de Gobierno Alcaldía Local de Ciudad Bolívar Cospacc, Junta Administradora Local Ciudad Bolívar.

ICBF - Instituto Colombiano de Bienestar Familiar \& Programa de las Naciones Unidas para los Asentamientos Humanos (ONU-HÁBITAT) (2014). Talleves para promover la participación de niños, niñas $y$ adolescentes en el desarrollo urbano.

Kaztman, R. (2011). Infancia en América Latina: privaciones habitacionales y desarrollo de capital humano. Santiago de Chile: Naciones Unidas - CEPAL UNICEF. Link, B. G. \& Phelan, J. C. (2001). Conceptualizing stigma. Annual Review of Sociology, 27, 363-385. doi http:// dx.doi.org/10.1146/annurev. soc.27.1.363

Ministerio de ambiente, vivienda y desarrollo territorial (s.f.). Guía en la vivienda de territarias 38

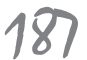




\section{tersitarias 38}

interés social. Serie guías de asistencia técnica para vivienda de interés social. Recuperado de http://www.minvivienda.gov.co/Documents/guia_asis_tec_ vis_1.pdf

Minujin, A., Bagnoli, V., Osorio, A.M. \& Aguado, L.F. (2015). Primera infancia cómo vamos: identificando desigualdades para impulsar la equidad en la infancia colombiana. Santiago de Cali, Colombia: Sello Editorial Javeriano.

Molano, F. (junio, 2015). El paro cívico de octubre de 1993 en Ciudad Bolívar (Bogotá): la formación de un campo de protesta urbana. Anuario colombiano de historia social y de la cultura, 42(1).

Molina, M. (2015). Representaciones discursivas de las personas en situación de pobreza urbana en notas periodisticas informativas publicadas en los principales diarios argentinos (Tesis doctoral, Universidad de Buenos Aires, Facultad de Filosofía y Letras, Buenos Aires, Argentina).

Moreno, L. (2015). Prólogo. En Los primeros años: el bienestar infantily el papel de las politicas públicas. Nueva York: Banco Interamericano de Desarrollo - BID.

Novoa, A., Boschc, J. Díaz, F., Malmusia, D., Darnelld, M. \& Trillad, C. (2014). Informe SESPAS: El impacto de la crisis en la relación entre vivienda y salud. Políticas de buenas prácticas para reducir las desigualdades en salud asociadas con las condiciones de vivienda. Gaceta Sanitaria, 28(1), 44-50.

Pautassi, L. \& Royo, L. (2012). CEPAL. Enfoque de derechos en las politicas de in- fancia: indicadores para su medición. Santiago de Chile: Naciones Unidas. Segovia, O. (2016). ¿Quién cuida en la ciudad? Oportunidades y propuestas en la comuna de Santiago (Chile). Serie asuntos de género, 132 (LC/L.4127), Santiago, Comisión Económica para América Latina y el Caribe (CEPAL).

Tarchópulos, D. \& Ceballos, O. (2003). Calidad de la vivienda dirigida a los sectores de bajos ingresos en Bogotá. Bogotá: Centro Editorial Javeriano.

Tarchópulos, D. \& Ceballos, O. (2005). Patrones arquitectónicos y urbanisticos en la vivienda dirigida a los sectores de bajos ingresos en Bogotá. Bogotá: Editorial Pontificia Universidad Javeriana.

Torres, C. (2010). Vivienda y hábitat en Colombia desde una perspectiva de derechos. Cuadernos electrónicos, 6.

Torres, J. (2016). Efectos de vecindario en Bogotá: Efectos de la segregación socioespacial en los logros educativos y laborales de la capital de Colombia. Centro de Estudios de la Construcción y el Desarrollo Urbano y Regional. Fondo editorial CENAC. Recuperado de http://www. cenac.org.co/index.shtml?apc=Il---$\& x=20158277$

Tuñón, I. \& González, M. S. (2013). Aproximación a la medición de la pobreza infantil desde un enfoque multidimensional y de derechos. Revista sociedad $y$ equidad, 5 .

UNICEF (2005). Estado mundial de la infancia 2005. La infancia amenazada. Nueva York: UNICEF. 
UNICEF - Fondo de las Naciones Unidas para la Infancia \& Oficina Regional para América Latina y el Caribe (2009). Estado mundial de la infancia. Edición especial. Conmemoración de los 20 años de la Convención sobre los derechos del niño. Nueva York.

Universidad de los Andes \& UNICEF (2012). Análisis de situación de la pobreza infantil en Colombia y revisión del Sistema de Protección Social sensible a las necesidades de los niños, niñas y adolescentes en Colombia. Bogotá: Escuela de Gobierno Alberto Lleras Camargo, Universidad de los Andes.

USAID (1993). Construyamos. Vivienda para el sector informal. Bogotá: Guadalupe.

Wacquant, L., Slater, T. \& Pereira, V. (2014). Estigmatización territorial en acción. Revista INVI, 29(82), 219-240. 
